\title{
Integralidade e construção de novas profissionalidades no contexto dos serviços substitutivos de saúde mental
}

\section{Integrality and the construction of new professionalities in the context of mental health substitutive services}

\author{
Elisabete Ferreira Mângia ${ }^{1}$, Melissa Muramoto²
}

MÂNGIA, E. F.; MURAMOTO, M. Integralidade e construção de novas profissionalidades no contexto dos serviços substitutivos de saúde mental. Rev. Ter. Ocup. Univ. São Paulo, v. 17, n. 3, p. 115-122, set./ dez., 2006.

RESUMO: As novas estratégias de cuidado em saúde mental, no contexto da Política Pública de Saúde brasileira, adotam uma nova forma de compreender e tratar os transtornos mentais que depende da estruturação de modelos assistenciais orientados pela perspectiva da integralidade, entendida aqui não apenas em referência à apreensão integral dos sujeitos, mas também aos novos valores e dispositivos técnicos. Este trabalho compõe a primeira fase do projeto de pesquisa "Estudo Colaborativo sobre a experiência da rede de serviços em saúde mental do município de Santo André: caracterizando os serviços, conhecendo resultados e desenvolvendo um experimento sobre ativação de rede social", iniciado em dezembro de 2005. Configurado como estudo qualitativo de inspiração etnometodológica, realizou entrevistas com usuários e técnicos, aplicação de questionários, observação participante em um Núcleo de Atenção Psicossocial, com funcionamento 24 horas. São apresentamos resultados parciais referentes ao Sub-Projeto A: Caracterização, funcionamento e estratégias da rede de serviços de saúde mental, com destaque para a discussão dos seguintes aspectos: a) integralidade e missão dos serviços substitutivos de saúde mental; b) necessidades formativas para a construção de novos perfis profissionais no campo da saúde mental e da reabilitação psicossocial; c) diretrizes para a construção de projetos terapêuticos na perspectiva da integralidade. Buscamos construir reflexões e indicações capazes de orientar a prática, a organização e a avaliação nos serviços de saúde mental, com especial interesse nos aspectos que compõe a relação entre profissionais e usuários e nas formas de construir, oferecer e avaliar as propostas e projetos terapêuticos. Nessas reflexões está presente o esforço para complexificar a compreensão do processo saúde-doença e assumir a integralidade em cada momento assistencial.

DESCRITORES: Serviços de saúde mental. Serviços comunitários de saúde mental. Transtornos mentais/ reabilitação. Assistência à saúde. Terapia ocupacional/psicologia.

\footnotetext{
${ }^{1}$ Prof. Dra. do Departamento de Fisioterapia, Fonoaudiologia e Terapia Ocupacional da FMUSP.

${ }^{2}$ Terapeuta Ocupacional, mestranda em Ciências da Reabilitação da FMUSP.

Endereço para correspondência: Rua Cipotânea, 51, Cidade Universitária, São Paulo, SP, CEP 05360-160.
} 


\section{INTRODUÇÃO}

A política de saúde mental no Brasil tem como eixos principais; a redução de leitos psiquiátricos, o maior controle sobre as internações, a organização de rede de serviços de saúde mental substitutivos e o reconhecimento dos direitos de cidadania das pessoas com transtornos mentais. Tais estratégias configuram uma nova forma de compreender e tratar os transtornos mentais que depende da estruturação de modelos assistenciais orientados pela perspectiva da integralidade, entendida aqui não apenas em referência à apreensão integral dos sujeitos, mas também aos novos valores e dispositivos técnicos (BRASIL, 2005, 2007).

Para a reabilitação psicossocial e mais especificamente para da terapia ocupacional, o desafio da inserção social de pessoas vulneráveis e o desenvolvimento de formas de convívio com a diferença exige transformações profundas nos modos de conceber o cuidado e organizar os serviços em confronto com as concepções e estratégias tradicionais o que implica na definição de novos perfis profissionais. Este trabalho compõe a primeira fase do projeto de pesquisa "Estudo Colaborativo sobre a experiência da rede de serviços em saúde mental do município de Santo André: caracterizando os serviços, conhecendo resultados e desenvolvendo um experimento sobre ativação de rede social", iniciado em dezembro de 2005, e que teve como objetivo geral, identificar as estratégias utilizadas na organização da rede de serviços e na atenção e reabilitação de pessoas com transtornos mentais, e seus resultados a partir do ponto de vista dos diversos atores envolvidos. Configurado como estudo qualitativo de inspiração etnometodológica, realizou entrevistas com usuários e técnicos, aplicação de questionários, observação participante em um Núcleo de Atenção Psicossocial, com funcionamento 24 horas. Apresentamos resultados parciais referentes ao SubProjeto A: Caracterização, funcionamento e estratégias da rede de serviços de saúde mental, com destaque para a discussão dos seguintes aspectos: a) integralidade e missão dos serviços substitutivos de saúde mental; b) necessidades formativas para a construção de novos perfis profissionais no campo da saúde mental e da reabilitação psicossocial; c) diretrizes para a construção de projetos terapêuticos na perspectiva da integralidade (GUIMARÃES, 1990; MINAYO, 2004).

\section{A crise dos serviços ou crise política?}

A participação no cotidiano e nas reuniões do serviço de alunos e técnicos da universidade, no contexto da estratégia da observação participante, além da elaboração coletiva de um diagnóstico de situação possibilitou a construção da reflexão que aqui apresentamos. Partimos do consenso, forjado nas discussões com os atores envolvidos, de que o serviço está em crise e para compreendermos esse processo consideramos importante a recuperação das diretrizes e estratégias da reforma psiquiátrica no Brasil e a reflexão sobre contribuições teóricas de autores que tem buscado formular caminhos para a construção de um modelo assistencial em Saúde Mental, substitutivo ao modelo tradicional. Sabemos que a construção de um novo modelo coloca em crise permanente não apenas o modelo que se quer superar mas também toda a cultura construída em torno da noção de doença mental/ periculosidade e incapacidade. É dessa crise que se espera o novo.

A "crise dos serviços", ou a crise de operatividade dos serviços, diante de novos objetivos e utopias, aparece como uma constante em inúmeras experiências nacionais e internacionais e parece fazer parte constitutiva do processo de construção das novas Políticas de Saúde Mental (SARACENO, 1998a; 1998b; 1999; OMS/PAS, 2001; CASTELFRANCHI et al., 1995).

Tal crise se configura a partir de alguns aspectos centrais:

- osserviçosnãorespondemadequadamenteas necessidades dos usuários com transtornos mentais severos;

- organizam-se a partir de estratégias fragmentadas e apresentam dificuldade na manutenção da continuidade terapêutica;

- não assumem plenamente a responsabilização pela demanda e tendem a colocar os usuários em um percurso continuo de trans-institucionalização;

- não desenvolvem uma política no território;

- não dispõe de projetos claros de reabilitação ou inserção no trabalho;

- mostram os limites das tecnologias médicopsiquiátrica, psicológica e reabilitativas tradicionais;

- fazem uso abusivo de fármacos;

- oferecem atividades desnecessárias;

- tendem a reproduzir a lógica de abandono e institucionalização em seu processo de trabalho;

Embora reconhecendo tais problemas, as equipes dos serviços tendem a atribuir a crise à agentes externos, à falta de recursos, aos recuos da política pública, à burocratização das relações, à falta de outros serviços de suporte. Apesar de muitos desses aspectos serem relevantes e, de fato, representarem prejuízo para a organização da política de saúde mental, existem aspectos 
MÂNGiA, E. F.; MURAmOTO, M. Integralidade e construção. Rev. Ter. Ocup. Univ. São Paulo, v. 17, n. 3, p. 115-122, set./dez., 2006.

fundamentais que estão no interior dos serviços e que deveriam ser considerados na avaliação dessa problemática, mas que dificilmente são assumidos pelas equipes. Nossa hipótese inicial é de que talvez faltem recursos técnicos, informações e parâmetros para criticar a própria prática e avançar nas propostas operativas. Assim, nosso objetivo aqui, a partir dos resultados encontrados em campo, vem a ser o de colocar em questão e definir aspectos que são considerados importantes, no contexto da literatura específica dessa área e do diálogo com as equipes de trabalho envolvidas neste projeto.

Nesse contexto o desafio da integralidade se torna um operador teórico e prático fundamental, pois vem sendo esse o horizonte buscado na construção de novos modelos assistenciais.

A noção de atenção integral à saúde, proposta na própria definição do Sistema Único de Saúde e inserida na Constituição brasileira, é um projeto político e ético ainda em construção (BRASIL, 1988, 1990). Concretizar um modelo assistencial ancorado em uma visão ampliada do processo saúde-doença e que toma como objeto a pessoa ou os grupos sociais, em seu contexto de vida e de acordo com as suas necessidades, depende de mudanças profundas em todos os aspectos implicados no trabalho de cuidado e promoção da saúde. Para a realização desse projeto, a perspectiva da integralidade deve estar presente em cada ação desencadeada pelo sistema, quer seja ela, local ou global, quer seja individual ou coletiva, e isso requer a transformação das tecnologias empregadas, da organização dos serviços, dos processos de trabalho em saúde e especialmente da formação dos profissionais e consequentemente, dos currículos universitários. Requer também mudanças nas relações estabelecidas entre profissionais de saúde e usuários.

Para Mattos (2001) "a integralidade não é apenas uma diretriz do SUS definida constitucionalmente. Ela é uma 'bandeira de luta', parte de uma 'imagem objetivo', um enunnciado de certas características do sistema de saúde, de suas instituições e de suas práticas que são consideradas (...) desejáveis. Ela tenta falar de um conjunto de valores pelos quais vale lutar, pois se relacionam a um ideal de uma sociedade mais justa e mais solidária".

Nesse sentido é que buscamos construir reflexões e indicações capazes de orientar a prática, a organização e a avaliação nos serviços de saúde mental, com especial interesse nos aspectos que compõe a relação entre profissionais e usuários e nas formas de construir, oferecer e avaliar as propostas e projetos terapêuticos. Nessas reflexões está presente o esforço para complexificar a compreensão do processo saúde-doença e assumir a integralidade em cada momento assistencial.

\section{Reforma psiquiátrica e as novas profissionalidades}

O processo da reforma passa por um momento importante e crítico de estruturação e consolidação que exige respostas inovadoras e criativas de seus protagonistas (BRASIL, 2007). Tais respostas terão um peso relevante nos destinos da nova Política de Saúde Mental e na definição do seu potencial de ruptura com a cultura manicomial, mais precisamente, com o paradigma da psiquiatria, que emergiu historicamente ancorado na abstração das definições sobre a doença mental, na invenção do hospital psiquiátrico como lócus da apreensão daqueles considerados perigosos e incapazes para os atos da vida social, de produção do saber sobre a doença e seu tratamento, na delegação de um novo tipo poder aos especialistas (a privação de liberdade mediada pela relação de tutela) (CASTEL, 1978; FOUCAULT, 1978, 1997).

Esse arranjo que produziu muito mais efeitos de violência e exclusão do que de cura, é que se tenta desmontar. Desinstitucionalização é muito mais do que fechar os manicômios e criar redes de serviços substitutivos, é inventar novas formas de convívio social com a diferença e isso implica em transformações profundas nos modos de pensar e fazer o cuidado, de definir o papel das instituições e especialmente coloca permanentemente em questão as diferentes concepções e estratégias utilizadas pelos profissionais, obrigando-nos também a nos reinventarmos (ROTELLI, 1999).

A construção de novos serviços e estratégias de cuidado tem se constituído no contexto do exercício das múltiplas profissionalidades. Há muitas formas de conceber e exercer as práticas multiprofissionais. Sabemos que no campo da atenção em saúde mental houve o desenvolvimento de perspectivas distintas que podem ser identificadas a partir de três modelos principais: o biomédico, o da escuta terapêutica e o do auxílio social (CASTEL, 1988; SARACENO et al., 1999). Já os modelos de reabilitação desenvolvidos no mesmo período são fortemente centrados no treino de habilidades sociais e/ ou laborais, ou no treinamento de famílias para sobreviverem aos transtornos mentais severos (SARACENO, 1999).

Ocorre que tais modelos, quando praticados de forma compartimentalizada e em distintos serviços assistenciais, que normalmente não mantêm relações entre si, além de se mostrarem insuficientes diante das necessidades das pessoas, trazem riscos importantes, sendo o maior deles o 
abandono dos sujeitos que não se adequam as ofertas e transitam permanentemente no circuito da transinstitucionalização ou sequer tem acesso a algum recurso (ROTELLI, 1999; DELL'ACQUA; MEZZINA, 1991).

Tais efeitos podem ser observados em muitos processos de reforma e constituem um enorme problema a ser enfrentado em nossa experiência.

Outra questão relevante que vem sendo colocada, diz respeito ao exercício ritual de certas formas de cuidado que não revertem em benefícios reais para a pessoa, nem interfere em sua qualidade de vida. Nas palavras de Saraceno (1999), mantêm os usuários "entretidos", reproduzindo aspectos da cultura manicomial. Saraceno (1999) provoca e ao mesmo tempo convoca todas as categorias profissionais a refletirem sobre as formas de entretenimento e nos incita ao "fim do entretenimento" como o principal desafio a ser enfrentado pelas práticas assistenciais e de reabilitação (CASTELFRANCHI et al., 1995).

O que fazer diante de processos que ao buscarem superar o paradigma psiquiátrico produziram efeitos indesejáveis? Como superar os resultados inesperados das práticas profissionais? Como renovar o potencial de respostas possíveis de serem agenciadas pelos profissionais de saúde? Como desenvolver modelos inovadores que produzam os efeitos desejados pelo processo da reforma: evitar o circuito de institucionalização, violência, supressão de direitos e abandono?

Desejamos inventar projetos de vida e de saúde e contribuir para a melhoria da qualidade de vida das pessoas, validar o direito á diferença, promover o acesso aos direitos sociais, dotar de sentido os projetos assistenciais e de reabilitação. Ao mesmo tempo, tentamos influir nos processos de educação e formação profissional. Desejamos criar processos de saber-fazer mais integrados e interativos e que no limite, sejam capazes de superar as barreiras entre as diferentes disciplinas, o conhecido desafio da interdisciplinariedade.

Parece impossível nos relacionarmos com tantas variáveis, mas ao mesmo tempo, temos que admitir que a emancipação do campo da saúde mental implica em mudanças em todos os seus componentes. Dessa forma, estamos todos em crise e temos que ser parceiros na superação dessa crise, temos que reinventar e dotar de sentido nossas profissionalidades e assim também, nos desinstitucionalizarmos (ROTELLI, 1999).

\section{Novas estratégias de cuidado: pressupostos para a construção serviços e projetos terapêuticos}

Reconhecer e definir projetos alternativos no campo da saúde mental se coloca, então, como o grande desafio das últimas décadas, as lentas mudanças no campo formativo não têm acompanhado, nem tampouco respondido adequadamente, aos desafios cotidianos enfrentados pelos novos serviços de saúde mental.

Para Saraceno (1998a,b, 1999), a separação entre sujeitos e contextos fundou a história da psiquiatria e a tarefa da reabilitação psicossocial seria superar essa separação ao considerar um terceiro elemento que é o serviço de saúde mental, como contexto de trocas, negociação e construção de projetos terapêuticos que se coloquem como elementos ativos na reconstrução e construção de percursos reais de vida.

Os recursos oferecidos pelos serviços, suas características materiais, organizativas e o estilo do trabalho da equipe são considerados variáveis importantes na evolução da enfermidade mental e juntamente com os recursos individuais do paciente e do seu contexto social e familiar desempenham um papel fundamental no sucesso ou fracasso de um tratamento. Essas variáveis, que durante muito tempo foram relegadas ao segundo plano, e não apenas aquelas classicamente consideradas "fortes" pela prática psiquiátrica (idade, diagnóstico e história da enfermidade), hoje são identificadas como as que realmente importam para o êxito das intervenções (SARACENO, 1999).

Nessa direção e ancorado em estudos que nas últimas décadas afirmaram a importância do contexto e da complexidade dos sujeitos, que não podem mais ser reduzidos a uma categoria diagnóstica, Saraceno (1999, p. 94) propõe, também, uma importante inversão na lógica de pensar os tratamentos ao sugerir a adoção de uma práxis que ao reconhecer os recursos de cada sujeito assuma o papel de acompanhá-lo na "construção de espaços de negociação".

Assumir que só a construção de espaços de troca pode gerar novas relações, implica no desenvolvimento de projetos terapêuticos inovadores, que adotem uma atitude crítica em relação aos tratamentos tradicionais farmacológicos, psicoterapicos ou reabilitativos centrados na remissão dos sintomas e no desenvolvimento de habilidades (quer sejam psicoafetivas, comunicacionais ou práticas), e que apostam na capacidade dos sujeitos de exercitarem ou transferirem para a vida real o que "aprenderam" nos espaços terapêuticos e/ou psicoterápicos individuais ou grupais, oferecidos pelos 
serviços. Nessa tradição, que caracteriza a maior parte dos serviços de tipo ambulatorial e na qual a maior parte dos profissionais de saúde mental ainda é formada, há uma clara dissociação entre o contexto de tratamento e o contexto de vida da pessoa e a oferta de cuidados se dá preferencialmente por meio de ofertas pré definidas e compartimentalizadas, de acordo com as diversas especialidades profissionais que operam com pouca ou nenhuma interação ou interatividade. As ofertas fragmentadas resultam na concepção de projeto terapêutico como somatória de diferentes procedimentos, desprovidos de um sentido claro para o paciente (DELL'ACQUA; MEZZINA, 1990; SARACENO, 1998b).

A adoção de uma nova compreensão técnica e ética da prática terapêutica dá consistência a construção do direito de cidadania como eixo prioritário e indissociável dos projetos terapêuticos destinados aos pacientes com transtornos mentais severos.

"Somente o cidadão pleno poderá exercitar as suas trocas, enquanto o cidadão partido ao meio não saberá o que fazer com as aquisições, com as habilidades relacionais, uma vez que não haverá direito nem acesso ao exercício de relações ou estas se darão como relações desprovidas da materialidade que as torna reais" (SARACENO, 1999, p. 94).

Para Rotelli (1993), o trabalho da saúde mental deveria ser o de habilitar e reabilitar na perspectiva da emancipação dos sujeitos em relação ao acesso aos direitos de cidadania, compreendidos em suas três dimensões: os direitos político, jurídicos e sociais. De acordo com este autor, reabilitar significa "Construir (reconstruir) acesso real aos direitos de cidadania, ao exercício progressivo dos mesmos, a possibilidade de vê-los reconhecidos e efetivados e a capacidade de praticá-los"

Nessa perspectiva são pilares que devem orientar a organização do processo de trabalho:

\section{OAcolhimento}

A construção de um novo modelo de atenção requer a avaliação crítica sobre o acesso e a possibilidade de ingresso dos usuários, familiares e cuidadores ao serviço, que implica na redefinição de procedimentos muitas vezes parcializados entre a recepção, triagem, acolhimento, ambiência e atendimento pontual de situações cotidianas, imprevistas ou mesmo àquelas consideradas críticas (MÂNGIA et al., 2002).

A separação dos procedimentos de acolhida muitas vezes prolonga demasiadamente a construção de respostas iniciais, fragiliza a responsabilização do serviço sobre a pessoa e suas necessidades, fazendo-a transitar por inúmeros interlocutores com pouca ou nenhuma responsabilidade sobre suas necessidades mais imediatas, nem tampouco sobre a possibilidade de construção de um projeto terapêutico. Burocratiza a relação inicial com a pessoa, podendo criar momentos de desgaste desnecessários tanto para a pessoa como para a equipe (MÂNGIA, 2002; MÂNGIA et al., 2002).

Essa reflexão nos leva a reconsiderar as estratégias tradicionalmente utilizadas em muitos serviços de saúde e propor que o acesso ao serviço e a possível tomada de responsabilidade sobre a pessoa possa ser configurada por meio de um processo mais ágil e humanizado, mais sensível à demanda no momento em que ela se apresenta configurada concretamente na busca do atendimento e formulação de sua necessidade.

Dessa forma, se coloca como adequado e possível reunir no procedimento de acolhimento tudo o que vem sendo fragmentado no processo de acesso ao serviço e atendimento de situações emergenciais e/ou cotidianas. Propõem-se enfim, um processo de acesso ao tratamento que seria iniciado a partir de um primeiro atendimento, conduzido por equipe responsável (de acordo com a lógica organizativa do serviço) e realizado assim que a pessoa busca a ajuda do serviço. Essa equipe assumiria imediatamente a responsabilidade pela formulação das primeiras respostas, dando início assim a construção de um projeto terapêutico.

Realizar o acolhimento quando a pessoa busca ou expressa necessidade rompe com a lógica da organização burocrática do serviço e responde adequadamente à necessidade da demanda com e quando esta se apresenta, isso traz profundas mudanças para o serviço, pois implica em inúmeros níveis de redefinição da oferta do serviço e da própria organização do trabalho técnico. Responder mais prontamente a demanda redefine competências e torna o serviço mais plástico e flexível ao mesmo tempo em que exige aumento do potencial de enfrentamento de situações diversificadas e concretas (MÂNGIA et al., 2002).

\section{A compreensão sobre a pessoa e doença}

A singularidade do sofrimento mental o coloca num patamar bem diverso daquele formulado pelo sujeito ao reconhecer alguma patologia ou mal estar orgânico e significar a sua enfermidade.

Se no processo dos acometimentos da saúde em geral sabemos que a possibilidade de formular a queixa, viver e significar o processo está carregada de conteúdos subjetivos e se constitui enquanto uma construção cultural, no que se convenciona assumir como doença mental quase não existe a possibilidade do reconhecimento 
MÂnGiA, E. F.; MURAMOTO, M. Integralidade e construção. Rev. Ter. Ocup. Univ. São Paulo, v. 17, n. 3, p. 115-122, set./dez., 2006.

da patologia propriamente dita, em termos do modelo clínico (anátomo-fisiológico) que orienta o pensamento biomédico. Não há a localização corporal, tecidual ou mesmo celular do sintoma e a conseqüente ação direta sobre essas concretudes, previstas pelo modelo clínico, não se realiza propriamente. Fazer cicatrizar ulceras ou lesões em tecidos, retirar tumores, reduzir fraturas, etc, tem certamente uma objetividade muito distinta daquela considerada puramente subjetiva e objetivada apenas nas diferenças comunicativas e comportamentais presentes na identificação e categorização do transtorno mental (FOUCAULT, 1980; SARACENO, 1998b; RABELO et al., 1999).

O fato é que, pacientes e técnicos se vêem às voltas com o imponderável configurado no sintoma e/ou queixas familiares, que levam os pacientes ao serviço, e na quase ausência de conhecimentos que assegurem a cura de tais transtornos. Ocorre que o processo vivido pela pessoa, tem no sintoma um elemento desencadeador de múltiplas perdas e desorganizações e reside muito mais nesses aspectos a configuração do campo de necessidades que leva a pessoa ao serviço (DELL'ACQUA; MEZZINA, 1990, 1991).

Dessa forma, as queixas são formuladas a partir da percepção de múltipos aspectos da desorganização do viver: nas relações familiares, na perda de vínculos, na fragilização das redes sociais e de suporte, na precarização do habitar e do poder de manter a existência concreta a partir de algum tipo de inserção laboral, dentre outros. Deve-se considerar que a doença mental é objetivada apenas na expressão de comportamentos disfuncionais ou seja, ganha visibilidade apenas quando se expressa no âmbito relacional e é aferida pelo grupo que constitui o entorno relacional da pessoa (RABELO et al., 1999).

Assim, a possibilidade de intervenção, em confronto com o frágil conhecimento teórico sobre os transtornos mentais severos e no contexto dos limites atuais das terapêuticas, pode ser configurada, mais positivamente, a partir do enfrentamento dos múltiplos aspectos necessários para a produção e reprodução objetiva e subjetiva da vida da pessoa. O debate contemporâneo sobre as novas estratégias de reabilitação psicossocial tem definido um território seguro de investimentos - a vida concreta da pessoa - que para ser alcançado exige a redefinição do processo de trabalho dos serviços e equipes e o deslocamento da ação terapêutica para os contextos reais nos quais transcorre a vida da pessoa, o que impõe o abandono dos contextos artificiais configurados pelos atendimentos que ocorrem exclusivamente no espaço físico dos serviços, e das práticas de entretenimento tão usuais hoje nos serviços de saúde mental (DELL'ACQUA; MEZZINA, 1990, 1991; SARACENO, 1999).

É fundamental que se reflita sobre essas questões para que de possa diferenciar os procedimentos a serem desenvolvidos e ao mesmo tempo, torná-los realmente úteis às pessoas. $\mathrm{O}$ que se faz e o como se faz num serviço de saúde mental deve se configurar como algo diferenciado, mas ao mesmo tempo produtivo, para não corremos o risco de entretemos os usuários com o nosso não saber.

O diagnóstico psiquiátrico ou psicológico consiste em abstrações a partir de arranjos sintomatológicos genéricos e que, no geral, conduzem ao uso de fármacos que apenas cumprem o papel de tentarem reduzir a expressão de tais sintomas - mas não se relacionam a uma expectativa de ação terapêutica de outro tipo.

Assim, qualificar um serviço de saúde mental consiste em conjugar recursos que redefinam os limites da ação clínica em seu sentido mais estrito e ao mesmo tempo, produzam respostas mais amplas e diversificadas dirigidas às pessoas e suas necessidades para o viver e não mais às abstrações diagnósticas ou psicologizantes ou ao combate dos sintomas isoladamente.

\section{A compreensão interativa: pessoa - contexto - história e recursos}

$\mathrm{A}$ atenção às pessoas com transtornos mentais severos e persistentes deve estar pautada na possibilidade de construção de projetos terapêuticos preocupados em promover o aumento dos fatores de proteção e a redução dos fatores de risco, que vulnerabilizam a pessoa e muitas vezes levam as recaídas ou trazem dificuldade na superação dos momentos críticos. A identificação desses fatores é fundamental para a condução de um adequado processo de oferta de respostas. É importante ressaltar que tal construção deve levar em conta a pessoa em seu contexto e na dimensão da construção/reconstrução de sua história (DELL'ACQUA; MEZZINA, 1990; CASTELFRANCHI et al., 1995; SARACENO, 1999).

Para tanto a intervenção deve assumir aspectos muitas vezes considerados "extra clínicos" pela cultura biomédica tradicional e ainda hegemônica. Sabemos que uma nova postura terapêutica implica também no decentramento da doença e da pessoa vista individualmente, como responsável pela sua falência biológica, psicológica ou social para um agenciamento relacional entre pessoa, contexto e recursos que estão fora da pessoa, mas que podem agir em seu favor. Não se pensa apenas em recursos materiais necessários para qualquer um de nós, mas também nos recursos sociais, culturais, relacionais, etc, capazes de reconstruir histórias e enriquecer contextos de vida 
muito empobrecidos, não só pelas carências de ordem material, mas especialmente pela vulnerabilidade de suportes sócio-relacional.

\section{A integralidade na prática}

Como nos ensina Mattos (2004):

"Defender a integralidade é defender, antes de tudo, que as práticas em saúde no SUS sejam sempre intersubjetivas, nas quais os profissionais de saúde se relacionem com os sujeitos e não com objetos. Práticas intersubjetivas envolvem necessariamente uma dimensão dialógica. Isso confere ás práticas de saúde um caráter de prática de conversação, na qual nós, profissionais de saúde, utilizamos nossos conhecimentos para identificar as necessidades de ações e serviços de saúde de cada sujeito com o qual nos relacionamos, para conhecer amplamente os conjuntos de ações que podemos por em prática".

Dessa forma, as dimensões e indicações aqui apresentadas, tem se mostrado operadores seguros nos diálogos sobre avaliação e construção de mudanças junto aos profissionais e estudantes participantes deste estudo. Acesso, garantido pela qualificação da prática de acolhimento é o ponto de partida para um novo tipo de vínculo e responsabilização que garanta aos usuários a construção de respostas complexas para enfrentar necessidades complexas. Para tanto, os profissionais devem estar aptos ao desenvolvimento de relações "sensíveis" e para a construção de projetos terapêuticos negociados e dotados de sentido.

As dimensões que caracterizam a atenção integral em saúde mental só podem ser construídas e realizadas quando todos os componentes das ações terapêuticas estejam orientados de acordo com uma lógica comum, que busca evitar a fragmentação dos sujeitos, das necessidades e das ações, ou seja que visa evitar a lógica da institucionalização e do abandono. Assim, a construção da integralidade configura não só um campo prático diferenciado, mas também uma nova ética do cuidado.

MÂNGIA, E. F.; MURAMOTO, M. Integrality and the construction of new professionalities in the context of mental health substitutive services. Rev. Ter. Ocup. Univ. São Paulo, v. 17, n. 3, p. 115-122, set./dez., 2006.

\begin{abstract}
S: The new strategies of care in mental health, in the context of Public Politics of Brazilian health, adopt a new way to comprehend and to treat the mental diseases, that depends on the structure of assistance's models oriented by the perspective of integrality, understood here not only in reference to the integral apprehension of the individuals, but also in reference to the new values and technical appliance. This paper is part of the first phase of the research project "Collaborative Study about the experience of the mental health services' net at the city of Santo André: characterizing the services, finding results and developing an experiment about social network activation", started in 2005, December. Configured as a qualitative study of ethnomethodological inspiration, actualized interviews with users and technicians, application of questionnaires, participative observation in a Psychosocial Care Center, opened 24 hours a day. We present partial results in reference to the subproject A: Characterization, functioning and strategies of the mental health services' net, standing out the discussion about the following aspects: a) the integrality and mission of the substitutive services in mental health; b) education needs to the construction of new professionals profiles in the mental health and psychosocial rehabilitation field; c) guidelines to the construction of therapeutics projects in the perspective of integrality. We tried to build reflections and indications able to orient the practice, the organization and evaluation in the mental health services, with special concern about the aspects that are part of the relationship between professionals and users and in the ways to build, offer and evaluate the proposals and therapeutic projects. In these reflections is present the effort to complexificate the comprehension about the health-disease process and to assume the integrality in every assistance moment.
\end{abstract}

KEY WORDS: Mental health services. Community mental health services. Mental disorders/ rehabilitation. Delivery of health caere. Occupational therapy/psychology. 
MÂngiA, E. F.; MURAMOTO, M. Integralidade e construção. Rev. Ter. Ocup. Univ. São Paulo, v. 17, n. 3, p. 115-122, set./dez., 2006.

\section{REFERÊNCIAS}

BRASIL. Ministério da Saúde. Secretaria de Atenção à Saúde. DAPE. Coordenação Geral de Saúde Mental. Reforma psiquiátrica e política de saúde mental no Brasil. In: CONFERÊNCIA REGIONAL DE REFORMA DOS SERVIÇOS DE SAÚDE MENTAL: 15 anos depois de Caracas. Brasília: OPAS; nov. 2005.

BRASIL. Ministério da Saúde. Secretaria de Atenção à Saúde/ DAPE. Saúde mental no SUS: acesso ao tratamento e mudança do modelo de atenção. Relatório de Gestão 2003-2006. Brasília; jan. 2007.

BRASIL. Constituição da República Federativa do Brasil, 1988. Brasília: Senado, 1988.

BRASIL. Lei n. 8080, de 19 de setembro de 1990. Dispõe sobre as condições para a promoção, proteção e recuperação da saúde, a organização e o funcionamento dos serviços correspondentes e dá outras providências.

CASTEL, R. A ordem psiquiátrica: a idade do ouro do alienismo. Rio de Janeiro: Graal, 1978.

CASTEL, R. Il decentramento del social, per la salute mentale, Rivista del Centro Studi Regionale per la Documentazione e Formazione Professionale nell'Ambito della Psichiatria del Friuli-Venezia Giulia, v. 1, p. 79-84, 1988.

CASTELFRANCHI, C.; HENRY, P.; PIRELLA, A. L' invenzione colletiva. Torino: Edizioni Gruppo Abele, 1995.

DELL'ACQUA, G.; MEZZINA, R. Resposta à crise: estratégia e intencionalidade da intervenção no serviço psiquiátrico territorial. In: DELGADO, J. (Org.). A loucura na sala de jantar. São Paulo, 1991.

DELL'ACQUA, G.; MEZZINA, R. Il Centro di Salute Mentale 24 ore come Comunità Terapeutica Allargata: La Riabilitazione e lo Sviluppo di una Rete Partecipativa. Estratto da:Riabilitazione Psicosociale in Psichiatria. Ruoli, Metodi, Strutture e Validazioni Atti del II Congresso Nazionale della Società Italiana di Riabilitazione Psicosociale, 8-9-10 Novembre 1990. Forte Spagnolo - L'Aquila. Disponível em: http://www.exclusion.net.

FOUCAULT, M. História da loucura na idade clássica. São Paulo: Perspectiva, 1978.

FOUCAULT, M. O poder psiquiátrico. In: FOUCAULT, M. Resumo dos cursos do Collège de France (1970-1982). Rio de Janeiro, Jorge Zahar Editor, 1997.

FOUCAULT, M. O nascimento da clínica. Rio de Janeiro: Forense Universitária, 1980.
GUIMARÃES, A. Z. Desvendando mascaras sociais. Rio de Janeiro: Francisco Alves, 1990.

MÂNGIA, E. F.; SOUZA, D. C.; HIDALGO, V. C. Acolhimento: uma postura, uma estratégia. Rev. Ter. Ocup. Univ. São Paulo, v. 13 , n. 1, p.15-21, 2002.

MÂNGIA, E. F. Contribuições da abordagem canadense "prática de terapia ocupacional centrada no cliente" e dos autores da desinstitucionalização italiana para a terapia ocupacional em saúde mental. Rev. Ter. Ocup. Univ. São Paulo, v. 13, n. 3, p. 127-134, 2002.

MATTOS, R. A. Os sentidos da integralidade: algumas reflexões acerca de valores que merecem ser defendidos. In: PINHEIRO, R.; MATTOS, R. A. de. Os sentidos da integralidade na atenção e no cuidado à saúde. Rio de Janeiro: IMS/ABRASCO, 2001. p. 39-64.

MATTOS, R. A. A integralidade na prática (ou sobre a prática da integralidade). Cad. Saúde Pública, Rio de Janeiro, v. 20, n. 5, p. 1411-1416, 2004.

MINAYO, M. C. S. O desafio do conhecimento: pesquisa qualitativa em saúde. São Paulo: Hucitec- Abrasco, 2004. p. 22.

ORGANIZAÇÃO MUNDIAL DA SAÚDE - OMS, Organização Panamericana da Saúde - OPAS . Relatório sobre a saúde no mundo - 2001. Saúde mental: nova concepção, nova esperança. Rio de Janeiro: Gráfica Brasil, Organização Mundial da Saúde, 2001.

RABELO, M.; ALVES, P.; SOUZA, I. Experiência de doença e narrativa. Rio de Janeiro: Ed. FIOCRUZ, 1999.

ROTELLI, F.; LEONARDIS, O.; MAURI, D. Desinstitucionalização, uma outra via. In: ROTELLI, F. et al. Desinstitucionalização. São Paulo: Hucitec, 1990.

ROTELLI, F. Per la normalità - taccuino di uno psichiatra negli anni della grande riforma scritti 1967-1998. Trieste: Scienza Nuova editore /Asterios Editore, 1999.

SARACENO, B.; ASIOLI, F.; TOGNONI, G. Manual de saúde mental. São Paulo: Hucitec, 1994.

SARACENO, B. A concepção de reabilitação psicossocial como referencial para as intervenções terapêuticas em saúde mental. Rev. Ter. Ocup. Univ. São Paulo, v. 9, n. 1, p. 1-44, 1998 a.

SARACENO, B. La cittadinanza come forma di tolleranza, Conferência. Rio de Janeiro, 1998b. Disponível em: http:// www.exclusion.net.

SARACENO, B. Libertando identidades. Da reabilitação psicossocial à cidadania possível. Belo Horizonte: Instituto Franco Basaglia/TeCorá, 1999. 